\title{
Copy Number Alterations of Depressed Colorectal Neoplasm Predict the Survival and Response to Oxaliplatin in Proximal Colon Cancer
}

\author{
Li-Chun Chang 1,2,3, Han-Mo Chiu ${ }^{1,2}{ }^{-}$, Bing-Ching Ho ${ }^{4}$, Min-Hsuan Chen ${ }^{4}$, Yin-Chen Hsu $5(\mathbb{D}$, \\ Wei-Tzu Chiu ${ }^{5}$, Kang-Yi Su ${ }^{5} \odot$, Chia-Tung Shun ${ }^{6}$, Jin-Tung Liang ${ }^{7,+}$, Sung-Liang Yu ${ }^{3,4,5,8,9,10,+}$ \\ and Ming-Shiang $\mathrm{Wu}^{1, *,+}$ \\ 1 Department of Internal Medicine, National Taiwan University Hospital, Taipei 100, Taiwan; \\ lichunchang@ntu.edu.tw (L.-C.C.); hanmochiu@ntu.edu.tw (H.-M.C.) \\ 2 Health Management Center, National Taiwan University Hospital, Taipei 100, Taiwan \\ 3 Graduate Institute of Clinical Medicine, National Taiwan University College of Medicine, Taipei 100, Taiwan; \\ slyu@ntu.edu.tw \\ 4 Centers of Genomic and Precision Medicine, National Taiwan University, Taipei 100, Taiwan; \\ f94424002@gmail.com (B.-C.H.); amp542chen@gmail.com (M.-H.C.) \\ 5 Department of Clinical Laboratory Sciences and Medical Biotechnology, College of Medicine, \\ National Taiwan University, Taipei 100, Taiwan; d04424009@ntu.edu.tw (Y.-C.H.); \\ chiuweitzu@hotmail.com (W.-T.C.); suky@ntu.edu.tw (K.-Y.S.) \\ 6 Department of Pathology and Forensic Medicine, National Taiwan University Hospital, Taipei 100, Taiwan; \\ ctshun@ntu.edu.tw \\ 7 Department of Surgery, National Taiwan University Hospital, Taipei 100, Taiwan; jintung@ntu.edu.tw \\ 8 Department of Laboratory Medicine, National Taiwan University Hospital, Taipei 100, Taiwan \\ 9 Institute of Medical Device and Imaging, College of Medicine, National Taiwan University, \\ Taipei 100, Taiwan \\ 10 Graduate Institute of Pathology, College of Medicine, National Taiwan University, Taipei 100, Taiwan \\ * Correspondence: mingshiang@ntu.edu.tw; Tel.: +886-2-23123456 (ext. 65043); Fax: +886-2-2341-2775 \\ + Co-senior authors.
}

Received: 5 May 2020; Accepted: 6 June 2020; Published: 10 June 2020

\begin{abstract}
Depressed colorectal neoplasm exhibits high malignant potential and shows rapid invasiveness. We investigated the genomic profile of depressed neoplasms and clarified the survival outcome and treatment response of the cancers arising from them. We examined 20 depressed and 13 polypoid neoplasms by genome-wide copy number analysis. Subsequently, we validated the identified copy number alterations (CNAs) in an independent cohort of 37 depressed and 42 polypoid neoplasms. Finally, the CNAs were tested as biomarkers in 530 colorectal cancers (CRCs) to clarify the clinical outcome of depressed neoplasms. CNAs in MYC, CCNA1, and BIRC7 were significantly enriched in depressed neoplasms and designated as the D-marker panel. CRCs with a D-marker panel have significantly shorter progression-free survival compared with those without $(p=0.012)$, especially in stage $\mathrm{I}(p=0.049)$, stages $\mathrm{T}_{1+2}(p=0.027)$, and proximal cancers $(p=0.002)$. The positivity of the D-marker panel was an independent risk factor of cancer progression (hazard ratio $(95 \%$ confidence interval) $=1.52(1.09-2.11)$ ). Furthermore, the proximal CRCs with D-marker panels had worse overall and progression-free survival when taking oxaliplatin as chemotherapy than those that did not. The D-marker panel may help to optimize treatment and surveillance in proximal CRC and develop a molecular test. However, the current result remains preliminary, and further validation in prospective trials is warranted in the future.
\end{abstract}

Keywords: colorectal cancer; CNAs; chemotherapy; MYC; CCNA1; BIRC7 


\section{Introduction}

The depressed colorectal neoplasm was first reported by Muto et al. in 1985 [1]. With technological progress and improved understanding, depressed neoplasms have been increasingly reported worldwide. These neoplasms exhibit a high malignant potential, with $33.3 \%$ to $66.6 \%$ of them harboring submucosal invasion at the time of diagnosis [2-6]. The endoscopic characteristics of depressed neoplasms are demonstrated in Figure S1e. Depressed neoplasms have a considerably higher risk of invasive cancer than flat $(1.3 \%)$ and polypoid $(0.18 \%)$ neoplasms [7]. Depressed neoplasms comprise $0.6 \%$ to $2.7 \%$ of all colorectal neoplasms, but their prevalence has increased to $33 \%$ to $39 \%$ of $\mathrm{T}_{1}$ colorectal cancer (CRC) [2-8]. The percentage could be as high as $60 \%$ when only considering cancer of less than $10 \mathrm{~mm}$ [7]. Currently, the molecular characteristics of depressed neoplasms remain elusive $[9,10]$. Given their rapid rate of growth, depressed neoplasms are considered to be high-risk lesions $[4,5,7]$, which in turn may potentially identify biomarkers from them to predict their prognosis and response to treatment in CRC.

Currently, the clinical management of CRC generally has not accounted for the primary site of neoplasm. However, the proximal and distal CRCs harbor different clinical and biologic characteristics. The proximal CRC is more likely to have the presence of $\mathrm{CpG}$ island methylator phenotype (CIMP), microsatellite instability (MSI), and BRAF mutation, in comparison with its distal counterparts. Previous studies have explored that the proximal CRC had a slightly favorable prognosis in stage II, but slightly unfavorable prognosis in stage III [11-13]. Besides prognosis, the response to chemotherapy is different between proximal and distal CRCs as well. The previous clinical trial has demonstrated that subjects with proximal CRC had inferior progression-free survival (PFS) after adjuvant chemotherapy or cetuximab, compared to those with distal cancer $[14,15]$. However, whether the survival and response to chemotherapy varies among proximal CRC with different molecular biomarkers was less addressed before. Oxaliplatin is one the most commonly used medications for advanced CRC as a first-line treatment. It remains unknown whether molecular biomarkers could stratify the prognosis and predict the response to chemotherapy with oxaliplatin as first-line treatment for proximal CRCs. Identification of molecular biomarkers to reach the abovementioned aims provides an opportunity to individualize the treatment for subjects with proximal CRC. Growing evidence has demonstrated the effectiveness of copy number alterations (CNAs) in predicting the prognosis of various cancers [16-18]. The Cancer Genome Atlas and other genomic landscape studies have explored specific CNAs in CRC, and these molecular alterations may have the potential to predict the prognosis as biomarkers $[19,20]$.

In this study, we investigated specific CNAs in depressed colorectal neoplasm and their potential impact as biomarkers on longitudinal clinical outcome and response to chemotherapy of CRCs, especially proximal CRC.

\section{Results}

\subsection{Identification of Biomarkers Specific to Depressed Reoplasms}

A total of 33 and 79 patients were included in the discovery and validation sets, respectively. The mean age and gender distribution in each category of neoplasm are listed in Table 1 . In the discovery set, 20 depressed and 13 polypoid neoplasms were examined. The mean tumor size of depressed and polypoid neoplasms was $12.1 \pm 6.1 \mathrm{~mm}$ and $28.1 \pm 9.3 \mathrm{~mm}$, respectively. Advanced histology was present in $50 \%$ of the depressed and $15.4 \%$ of the polypoid neoplasms. In the validation set, 37 depressed and 42 polypoid neoplasms were included. The mean tumor size of depressed and polypoid neoplasms was $24.3 \pm 10.6 \mathrm{~mm}$ and $21.0 \pm 11.3 \mathrm{~mm}$, respectively. Advanced histology was present in $97.3 \%$ of depressed and $28.6 \%$ of polypoid neoplasms. Although the sample collection was by time sequence, the depressed neoplasms in the validation set exhibited a higher percentage of advanced histology than those in the discovery set. Invasive cancer could be found in depressed neoplasms with small size, and some of them are demonstrated in Figure 1. 
Table 1. Demographic and clinical information.

\begin{tabular}{|c|c|c|c|c|}
\hline \multirow{2}{*}{ Clinical Information } & \multicolumn{2}{|c|}{ Discovery Set } & \multicolumn{2}{|c|}{ Validation Set } \\
\hline & D Type, $n=20$ & P Type, $n=13$ & D Type, $n=37$ & P Type, $n=42$ \\
\hline Male, $n(\%)$ & $14(70.0)$ & $7(53.8)$ & $26(70.3)$ & $30(71.4)$ \\
\hline Age, years (SD) & $66.5(9.0)$ & $58.5(11.7)$ & $66.5(12.5)$ & $65.7(12.7)$ \\
\hline \multicolumn{5}{|l|}{ Location, $n(\%)$} \\
\hline Proximal & $8(40.0)$ & $3(23.1)$ & $16(43.2)$ & $20(47.6)$ \\
\hline Distal & $10(50.0)$ & $9(69.2)$ & $12(32.4)$ & $13(31.0)$ \\
\hline Rectum & $2(10.0)$ & $1(7.7)$ & $9(24.3)$ & $9(21.4)$ \\
\hline Tumor size, mm (SD) & $12.1(6.1)$ & $28.1(9.3)$ & $24.3(10.6)$ & $21.0(11.3)$ \\
\hline \multicolumn{5}{|l|}{ Histology, $n(\%)$} \\
\hline Tubular adenoma & $8(40.0)$ & $3(23.1)$ & $1(2.7)$ & $9(21.4)$ \\
\hline Tubulovillous adenoma & $2(10.0)$ & $8(61.5)$ & $0(0)$ & $21(50.0)$ \\
\hline Advanced histology & $10(50.0)$ & $2(15.4)$ & $36(97.3)$ & $12(28.6)$ \\
\hline High-grade dysplasia & $3(15.0)$ & $2(15.4)$ & $21(56.8)$ & $9(21.4)$ \\
\hline Invasive cancer & $7(35.0)$ & $0(0)$ & $15(40.5)$ & $3(7.1)$ \\
\hline $\mathrm{G} 1 / 2$ & $7(35.0)$ & $0(0)$ & $15(40.5)$ & $3(7.1)$ \\
\hline $\mathrm{G} 3 / 4$ & $0(0)$ & $0(0)$ & $0(0)$ & $0(0)$ \\
\hline \multicolumn{5}{|l|}{ Morphology, $n(\%)$} \\
\hline 0-Ip & - & $8(61.5)$ & - & $23(54.8)$ \\
\hline 0 -Is & - & $5(38.5)$ & - & $19(45.2)$ \\
\hline 0-IIc & $5(25.0)$ & - & $2(5.4)$ & - \\
\hline 0-IIa+IIc & $10(50.0)$ & - & $31(83.8)$ & - \\
\hline 0-Is+IIc & $5(25.0)$ & - & $4(10.8)$ & - \\
\hline
\end{tabular}

D type: depressed neoplasm, P type: polypoid neoplasm.

The CNA analysis of depressed and polypoid neoplasms is shown in Figure S2A and 2B, respectively. The merged copy number pattern with the arm-level and copy number aberration across all chromosomes is presented in Figure S2C. Through testing formalin-fixed paraffin-embedded (FFPE) tissues, we have identified five significant arm-level changes (chr3, chr8, chr13, chr20, and chrX). The depressed neoplasms had a CNA gain in chr8, chr13, chr20, and chrX, and a CNA loss in chr3 in comparison with polypoid counterparts. Among them, the arm-level change at chr3 and chrX was a non-coding region, and no corresponding gene was identified. We matched the protein-coding gene with significant arm-level changes at chr8, chr13, and chr20, and the associated genes are listed in Table S1. On the basis of the nature of early malignant transformation and aggressively invasive potential in depressed neoplasms, we searched the potential corresponding genes associated with tumorigenesis, invasiveness, or metastasis of CRC by the arm-level change specific to a depressed neoplasm. Among these associated genes, searched by MetaCore Analytical Suite (GeneGo, Saint Joseph, MI, USA) and the GeneCards online database (www.genecards.org), MYC, CCNA1, and BIRC7 were identified to be the representatives at chr8, chr13, and chr20, respectively [21-24].

\subsection{Diagnostic Performance of the D-Marker Ranel}

We used MYC, CCNA1, and BIRC7 in combination as a D-marker panel for identifying depressed neoplasms, and tested the panel in the discovery set. The neoplasms that carried at least one alteration in the three genes were diagnosed as depressed neoplasms. The sensitivity, specificity, and accuracy of the D-marker panel for discriminating the depressed from the polypoid neoplasms and the arm-level changes in MYC, CCNA1, and BIRC7 in each case in the discovery set were calculated. (Table S2 and Figure S3). Subsequently, we validated the D-marker panel in the larger validation set, and the sensitivity, specificity, and accuracy for detecting depressed neoplasms increased to $75.7 \%, 83.3 \%$, and $79.7 \%$, respectively. Given the more advanced histology and larger sample size in the validation set, the accuracy of the D-marker panel was better in the validation set than in the discovery set. 


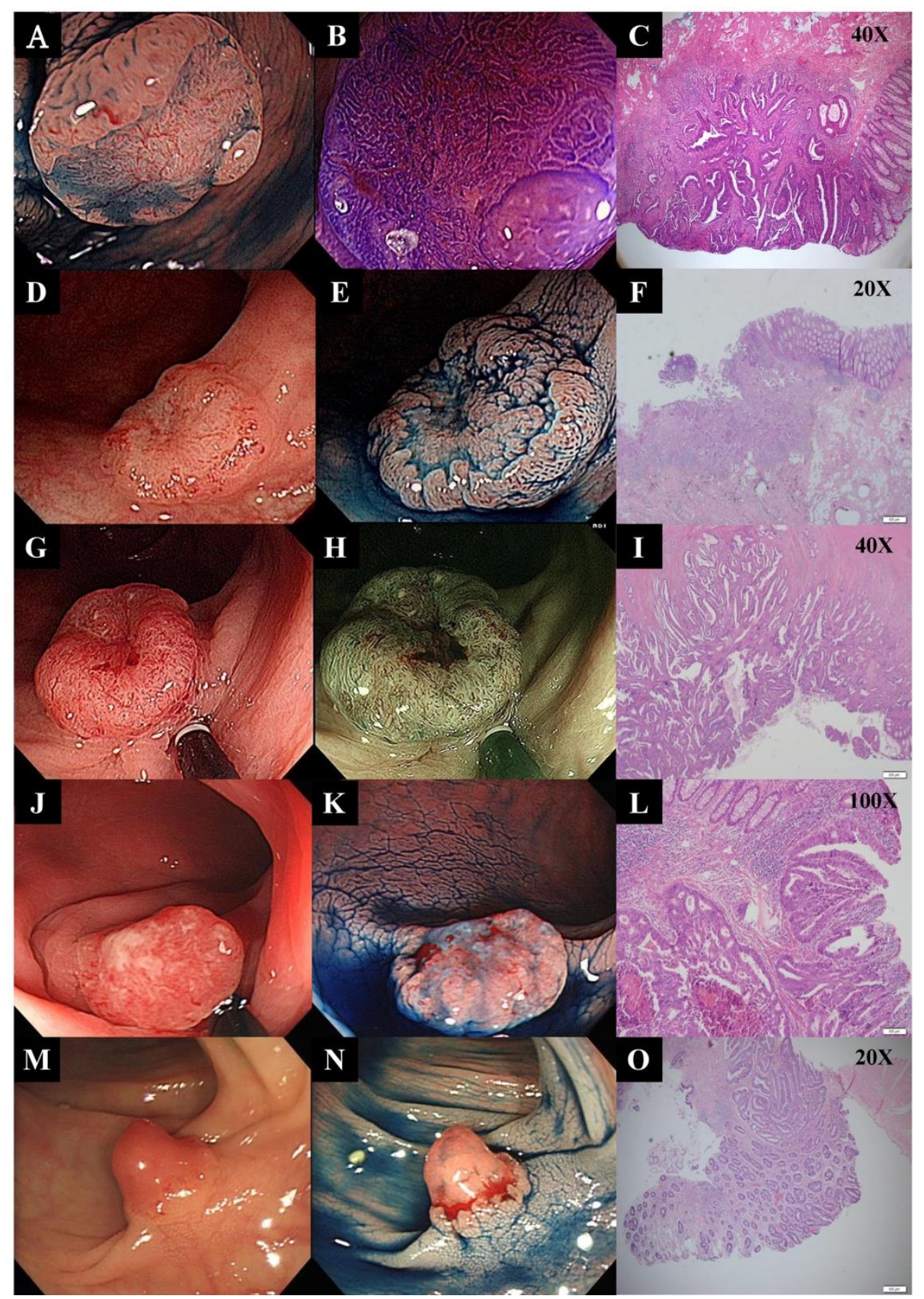

Figure 1. Depressed colorectal neoplasms harbor high malignant potential and presents with invasive cancer, even at a small size. The first depressed neoplasm is $8 \mathrm{~mm}$ in size and appears as 0-IIa + IIc in morphology (A,B). The adenocarcinoma presents with submucosal invasion $(1.8 \mathrm{~mm})$ in the depressed area $(\mathbf{C})$. The second depressed neoplasm is $8 \mathrm{~mm}$ in size and harbors invasive cancer with submucosal invasion. Moreover, one of the 22 regional lymph nodes is metastasized, and the pathological stage is T1N1aM0 (D,E,F). The third depressed neoplasm is $1.2 \mathrm{~cm}$ in size and 0-IIa + IIc in morphology $(\mathbf{G}, \mathbf{H})$. The adenocarcinoma in the depressed area invades into the muscular layer, and thereby its stage is T3N0M0 (I). The central depressed area starts to rise significantly when the cancer keeps growing $(\mathbf{J}, \mathbf{K})$, and eventually becomes protruded (0-Is + IIc) $(\mathbf{M}, \mathbf{N})$, which as a morphological appearance hints at deep submucosal invasion by cancer. Both the last two lesions are submucosal cancer $(\mathbf{L}, \mathbf{O})$, although their size is as small as $10 \mathrm{~mm}$. 


\subsection{Clinical and Molecular Characteristics of CRC with A Positive D-Marker Panel}

To evaluate the impact of a D-marker panel on CRC outcome, a total of 530 frozen CRC tissues were enrolled for testing the survival outcome, and their stage distribution was 170 (32\%) stage I, 134 (25\%) stage II, 120 (23\%) stage III, and 106 (20\%) stage IV. Two hundred and seventy-five (51.9\%) of them were male, and 177 (33.4\%) were anatomically proximal cancers.

From molecular point of view, a CRC with a positive D-marker panel was defined as a "depressed CRC". The depressed CRC was considered to be growing from the depressed neoplasm. The depressed CRCs accounted for $22.1 \%$ of all CRCs and $14.7 \%$ of the proximal CRCs. The distribution of depressed CRC in stages I, II, III, and IV was $18.2 \%, 23.9 \%, 20.0 \%$, and $28.3 \%$, respectively. The comparison of clinical information between depressed and conventional CRCs is listed in Table 2. Depressed CRCs were proximally located less frequently. KRAS, microsatellite instability-high (MSI-H), and CIMP-positivity were significantly $(p=0.018, p=0.0007$, and $p=0.018$, respectively) less common in depressed CRC than in conventional CRC.

Table 2. Comparison of depressed colorectal cancer (CRC) with conventional CRC.

\begin{tabular}{cccc}
\hline Clinical Information & $\begin{array}{c}\text { Depressed CRC } \\
\boldsymbol{n}=\mathbf{1 1 7}\end{array}$ & $\begin{array}{c}\text { Conventional CRC } \\
\boldsymbol{n}=\mathbf{4 1 3}\end{array}$ & $\boldsymbol{p}$-Value \\
\hline Mean age, years (SD) & $70.8(13.7)$ & $70.8(14.3)$ & 0.00 \\
Male gender, $n$ (\%) & $55(47.0)$ & $220(53.3)$ & 0.23 \\
Proximal location, $n(\%)$ & $26(22.2)$ & $151(36.6)$ & 0.0037 \\
Cancer stage distribution & & & \\
$\mathrm{T}_{1}, n(\%)$ & $13(11.1)$ & $61(14.8)$ & 0.32 \\
$\mathrm{~T}_{1+2}, n(\%)$ & $38(32.5)$ & $157(38.0)$ & 0.27 \\
Stage I, $n(\%)$ & $31(26.5)$ & $139(33.7)$ & 0.14 \\
Stage II, $n(\%)$ & $32(27.4)$ & $102(24.7)$ & 0.56 \\
Stage III, $n(\%)$ & $24(20.5)$ & $96(23.2)$ & 0.53 \\
Stage IV, $n(\%)$ & $30(25.6)$ & $76(18.4)$ & 0.084 \\
Molecular characteristics & & & \\
KRAS mutation, $n(\%)$ & $33(28.2)$ & $166(40.2)$ & 0.018 \\
BRAF mutation, $n(\%)$ & $3(2.6)$ & $21(5.1)$ & 0.25 \\
MSI-H, $n(\%)$ & $0(0)$ & $38(9.2)$ & 0.0007 \\
CIMP-positivity, $n(\%)$ & $8(6.8)$ & $63(15.3)$ & 0.018 \\
\hline
\end{tabular}

MSI-H: microsatellite instability-high, CIMP: CpG island methylation phenotype. Depressed CRC: colorectal cancer with positive D-marker panel; conventional CRC: colorectal cancer with negative D-marker panel.

\subsection{Survival Outcome of CRC with a Positive D-Marker Panel}

Depressed CRC had significantly more unfavorable PFS compared with conventional CRC $(p=0.012)$ (Figure 2). The unfavorable outcome of depressed CRC was especially found in proximally located CRC $(p=0.002)$, stage I CRC $(p=0.049)$, and $\mathrm{T}_{1+2} \mathrm{CRC}(p=0.027)$ (Figure $\left.2 \mathrm{C}, \mathrm{E}, \mathrm{G}\right)$ For overall CRC, depressed CRC with KRAS or BRAF mutations had the most unfavorable PFS, followed by CIMP-positivity, and then with D-marker alone in comparison with conventional CRC $(p=0.04$; Figure 3). The result of Cox regression is listed in Table 3. In the multivariable analysis (Model 1), the positivity of the D-marker panel remained an independent risk factor for cancer progression after adjusting for cancer differentiation, KRAS mutation, and BRAF mutation, and the adjusted hazard ratio (HR) (95\% CI) was 1.52 (1.09 to 2.11). Even after adjusting for the cancer stage, the positivity of the D-marker panel and cancer progression remained associated with marginal significance, and the adjusted HR (95\% CI) was 1.29 (0.92 to 1.80) (Model 2). Regarding $\mathrm{T}_{1+2}$ cancer, the positivity of the D-marker panel was an independent risk factor for cancer recurrence, and the adjusted $\mathrm{HR}$ (95\% CI) was 4.37 (1.05 to 18.26) after adjusting for the age, gender, and KRAS mutation (Table 4). The proximal CRC with a positive D-marker panel had a less favorable survival outcome, in terms of both overall survival (OS) and PFS, than those without a positive panel when taking chemotherapy with oxaliplatin as first-line chemotherapy. The $p$-values were 0.045 and 0.0065 , respectively (Figure 4 ). 
A

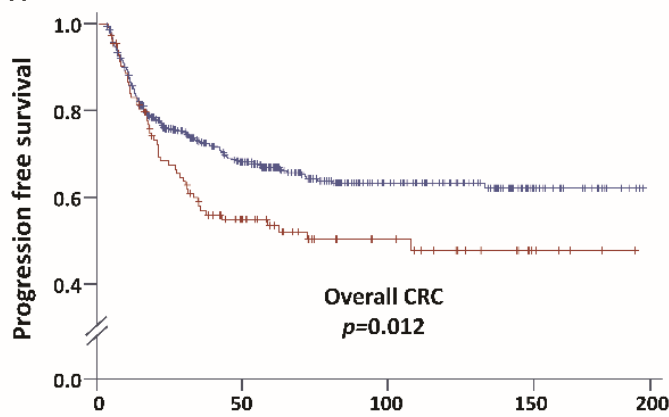

Conventional CRC 421

Depressed CRC 117

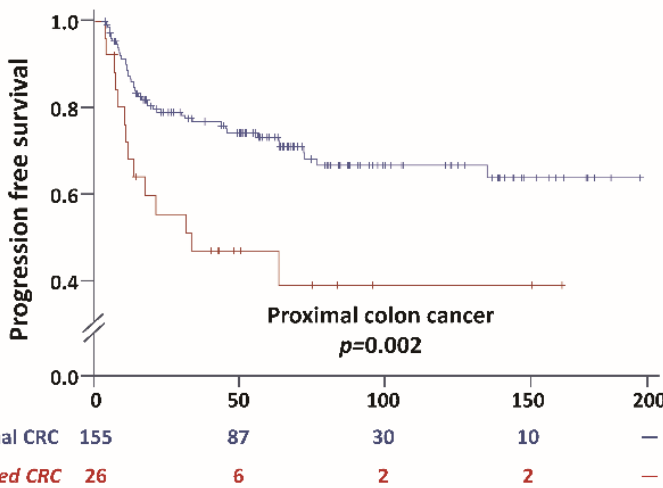

E

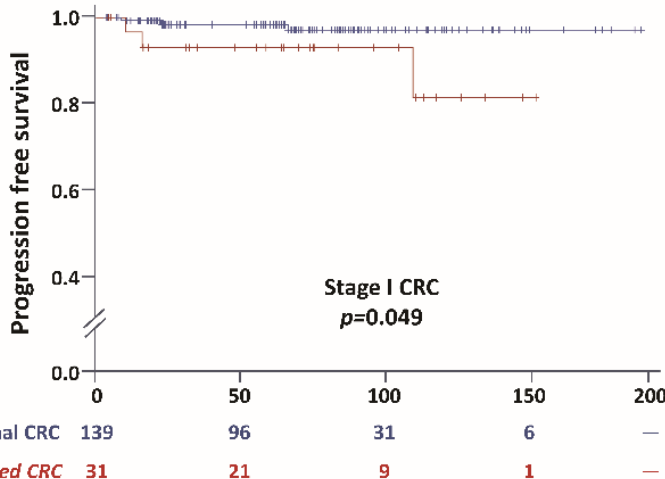

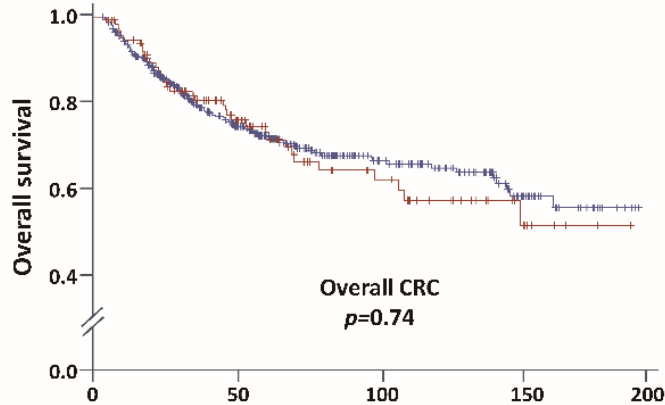

$438 \quad 234$

$120 \quad 60$



F



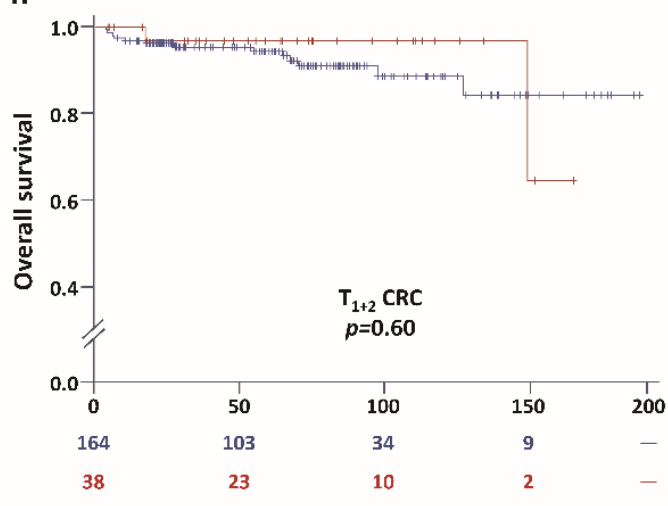

Follow-up period (month)

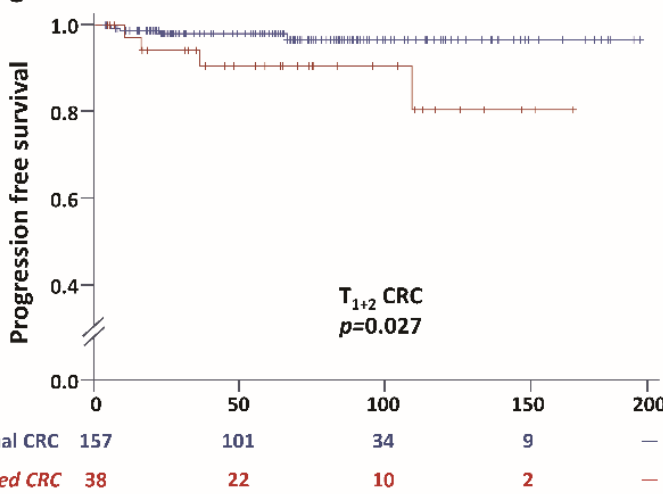

Follow-up period (month)

Figure 2. Depressed CRC associated with unfavorable survival compared with conventional CRC. The depressed CRC has an unfavorable progression-free survival (PFS) in comparison with the conventional CRC in overall CRC (A), proximal colon (C), early-stage (stage I) CRC (E), and small-sized cancer $\left(\mathrm{T}_{1+2}\right.$ cancer $)(\mathbf{G})$. The overall survival $(\mathrm{OS})$ is not significantly different between depressed CRC and conventional CRC with regard to overall $(\mathbf{B})$, proximal $(\mathbf{D})$, stage $\mathrm{I}(\mathbf{F})$, and $\mathrm{T}_{1+2}$ cancers $(\mathbf{H})$. 


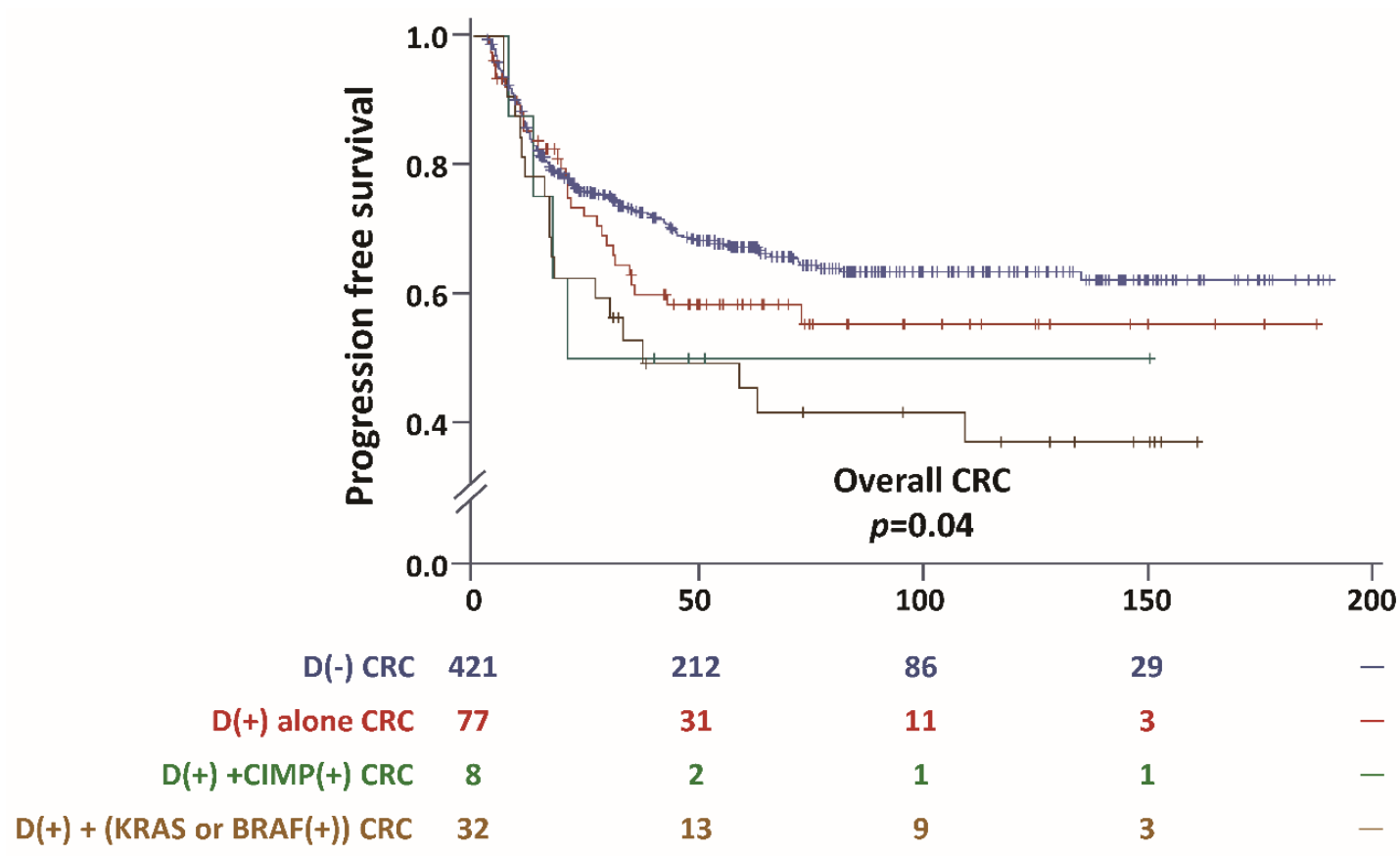

Follow-up period (month)

Figure 3. Comparison of progression-free survival (PFS) among depressed CRCs with various genetic changes. Depressed CRC has an unfavorable PFS compared to conventional CRC. The survival deteriorates further if KRAS or BRAF mutation presents, as well as if the CRC is CIMP-positive. $\mathrm{D}(-)$ : conventional CRC; $\mathrm{D}(+)$ : depressed CRC; CIMP(+): CIMP-positivity.

Table 3. Risk factor for tumor progression in CRC.

\begin{tabular}{|c|c|c|c|c|c|c|}
\hline \multirow{2}{*}{ Clinical Information } & \multicolumn{2}{|c|}{ Univariable } & \multicolumn{2}{|c|}{ Model 1} & \multicolumn{2}{|c|}{ Model 2} \\
\hline & HR & $95 \% \mathrm{CI}$ & aHR & $95 \% \mathrm{CI}$ & aHR & $95 \% \mathrm{CI}$ \\
\hline Age & 0.98 & 0.97 to 0.99 & 0.97 & 0.96 to 0.99 & 0.99 & 0.97 to 1.0 \\
\hline Gender, male vs. female & 1.23 & 0.93 to 1.64 & 1.47 & 1.09 to 1.97 & 1.26 & 0.93 to 1.7 \\
\hline Location, proximal vs. distal & 1.14 & 0.83 to 1.55 & & & & \\
\hline \multicolumn{7}{|l|}{ Cancer stage } \\
\hline I & 1 & & & & 1 & \\
\hline II & 5.35 & 2.20 to 13.0 & & & 5.07 & 2.08 to 12.38 \\
\hline III & 22.23 & 9.61 to 51.39 & & & 20.26 & 8.73 to 47.04 \\
\hline IV & 59.05 & 25.72 to 135.59 & & & 53.03 & 22.95 to 122.54 \\
\hline Positivity of D-marker panel & 1.47 & 1.07 to 2.01 & 1.52 & 1.09 to 2.11 & 1.29 & 0.92 to 1.80 \\
\hline \multicolumn{7}{|l|}{ Cancer differentiation } \\
\hline Low-grade & 1 & & 1 & & 1 & \\
\hline High-grade & 3.13 & 1.54 to 6.36 & 2.79 & 1.31 to 5.95 & 2.45 & 1.15 to 5.24 \\
\hline Mucinous & 2.86 & 0.71 to 11.60 & 3.58 & 0.87 to 14.72 & 1.06 & 0.26 to 4.38 \\
\hline KRAS mutation & 1.23 & 0.92 to 1.64 & 1.51 & 1.11 to 2.05 & 1.72 & 1.26 to 2.36 \\
\hline$B R A F$ mutation & 0.93 & 0.46 to 1.89 & 1.16 & 0.55 to 2.44 & 1.60 & 0.75 to 3.42 \\
\hline MSI-H & 0.24 & 0.09 to 0.65 & 0.22 & 0.08 to 0.60 & 0.46 & 0.17 to 1.26 \\
\hline CIMP-positivity & 0.86 & 0.55 to 1.34 & 0.92 & 0.58 to 1.47 & 0.77 & 0.48 to 1.22 \\
\hline
\end{tabular}

MSI: microsatellite instability; CIMP: CpG island methylation phenotype; HR: hazard ratio; aHR: adjusted HR. 
Table 4. Risk factors for cancer recurrence in $\mathrm{T}_{1+2}$ cancer.

\begin{tabular}{ccccc}
\hline \multirow{2}{*}{ Clinical Information } & \multicolumn{2}{c}{ Univariable } & \multicolumn{2}{c}{ Multivariable } \\
\cline { 2 - 5 } & HR & $\mathbf{9 5 \%}$ CI & aHR & 95\% CI \\
\hline Age & 1.00 & 0.95 to 1.06 & 1.01 & 0.96 to 1.06 \\
Gender & 1.14 & 0.29 to 4.56 & 1.44 & 0.34 to 6.07 \\
Location, proximal vs. distal & 1.34 & 0.27 to 6.64 & & \\
Positivity of D-marker panel & 4.22 & 1.05 to 16.90 & 4.37 & 1.05 to 18.26 \\
KRAS mutation & 1.05 & 0.25 to 4.40 & 1.09 & 0.25 to 4.75 \\
\hline
\end{tabular}

A

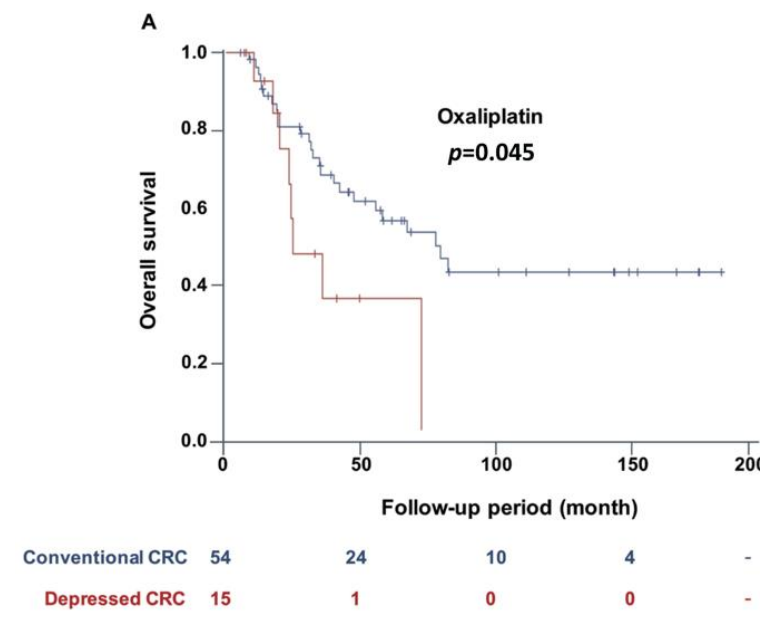



Figure 4. Comparison of survival outcome between proximal CRC with and without a positive D-marker panel when using oxaliplatin as first-line chemotherapy. Proximal CRC with a positive D-marker panel had a worse response to chemotherapy with oxaliplatin as a first-line agent. Compared to those without D-marker, proximal CRC with a positive D-marker panel had an unfavorable overall survival (A) and progression-free survival (B). The $p$-values were 0.045 and 0.0065 , respectively.

\section{Discussion}

In the present study, we identified three genomic alterations of CNAs, which are specific to depressed neoplasms, namely MYC, CCNA1, and BIRC7. When applying the D-marker panel, depressed CRC accounted for $22.1 \%$ of the CRCs. This proportion is consistent with previous estimations in observational studies or modeling studies [25,26]. Importantly, CRCs with these CNAs had a significantly unfavorable PFS compared to those without. The unfavorable PFS was more pronounced in stage I, $\mathrm{T}_{1+2}$, and proximal cancers. Moreover, proximal CRC with the biomarkers had a worse PFS and OS than those without. Those findings help understanding of the heterogeneity of colorectal carcinogenesis, therefore contributing to the future development of screening or surveillance biomarkers and the establishment of tailored treatment strategies. Moreover, the result may help to stratify the risk of recurrence and predict the response to chemotherapy in proximal CRC.

The present study demonstrated that the proximal CRC with a D-marker panel had a more unfavorable PFS and a worse response to oxaliplatin as first-line chemotherapy. The findings can be applied to stratify the risk of recurrence of proximal CRC, and thereby to provide individualized surveillance accordingly. Moreover, the selection of chemotherapy can be tailored by the present results. Oxaliplatin should be avoided as first-line treatment in proximal CRC with biomarkers. In addition, these results may help in customizing the treatment of early cancer and post-resection surveillance. High-risk $\mathrm{T}_{1}$ cancer, which has unfavorable histology, requires surgery because of the risk of lymph node metastasis (LNM) [27]. In contrast, low-risk $T_{1}$ cancer can be cured through endoscopic resection alone. Nevertheless, nearly $90 \%$ of $\mathrm{T}_{1}$ cancers, which are defined as high-risk based on 
current histologic criteria, do not have LNM [28]. The molecular diagnosis may compensate for the insufficiency of the histological criteria and aid in clinical decision-making regarding best therapy (surgery or endoscopic resection) [29]. Moreover, the current guidelines for the treatment of stage I CRC recommend no additional therapy after resection. It remains unclear whether adjuvant therapy is beneficial for stage I cancer carrying a higher risk of recurrence. In our study, early CRCs, either stage I or $\mathrm{T}_{1+2}$, with a positive $\mathrm{D}$-marker panel had a significantly higher risk of recurrence. This finding may help to stratify early-stage CRC and tailor its treatment and surveillance.

CRC progresses via different pathways. Older studies from Japan found that some cancers arising from depressed neoplasms do not exhibit an overt adenomatous component [7]. The researchers hypothesized such cancers' progress via de novo carcinogenesis. The present study attempts to clarify the biologic signature and potential clinical relevance of CRC that arises from depressed neoplasms. It has been shown that depressed neoplasms have a rapid progression. Previous observational studies have found that $22.9 \%$ to $39 \%$ of $\mathrm{T}_{1}$ or $\mathrm{T}_{2}$ cancer is depressed cancer $[7,8,25]$. In a modeling study, the depressed pathway is estimated to contribute to $30 \%$ of all CRCs [26]. The present study helps to understand the cancer arising from the depressed neoplasm from the molecular aspect.

The understanding of molecular mechanisms that underlie the carcinogenesis in depressed neoplasm is largely unknown. The present study demonstrates that MYC, CCNA1, and BIRC7 alterations are significantly enriched in depressed neoplasms. MYC is crucial in cell cycle progression, apoptosis, and cellular transformation. The up-regulation of $M Y C$ could drive oncogenic transformation [30]. CCNA1 might control the cell cycle, and contributes to cancer invasion and metastasis [23]. BIRC7 is involved with cell proliferation, invasion, and migration [31]. Therefore, BIRC7 has be considered to play a critical role in the development of CRC metastasis [24]. Taken together, MYC might initiate the carcinogenesis of depressed neoplasms at the early stage, and subsequently, CCNA1 and BIRC7 might promote its invasiveness and further migration. This might explain the aggressive presentation of depressed neoplasms.

There may be three reasons to explain the unfavorable outcome of depressed neoplasms. First, the increased risk of recurrence in either stage $\mathrm{I}$ or $\mathrm{T}_{1+2}$ stages indirectly implies that depressed neoplasms not only develop into invasive cancer despite being a small size or early-stage, but also spread out concurrently. Second, depressed neoplasms have a subtle endoscopic appearance, and early diagnoses are challenging. CRCs with MSI-H have a favorable survival outcome compared to those with microsatellite instable-low/microsatellite stable (MSI-L/MSS) [32]. The presence of D-maker panel and MSI-H were mutually excluded. The rare occurrence of MSI-H in depressed CRCs may lead to an unfavorable outcome.

Our study had several strengths. This study is the first to explore the molecular changes specific to depressed neoplasms, and subsequently to investigate survival outcomes through those specific markers. The sample size of the depressed neoplasm is the largest available, to our best knowledge. Various experimental methods have been used to test molecular changes in depressed neoplasms. The present study first investigated the genome-wide CNAs in depressed neoplasms. Furthermore, all neoplasms included in the discovery cohort were benign or at the $\mathrm{T}_{0}$ or $\mathrm{T}_{1}$ stage. The three genetic changes may act as a significant driver in the initiation of carcinogenesis. In contrast, almost all lesions in the discovery cohort achieved curative treatment without recurrence because of their early-stage nature. Therefore, the current study could not explore the survival outcome of these lesions. This limitation, however, has been overcome by validating the D-marker panel in advanced CRC. We applied selective dye-based chromoendoscopy for the detection and diagnosis of depressed colorectal neoplasms, thereby improving the accuracy of our data [33]. The significant comorbidity of the enrolled subjects was lacking. Therefore, the survival outcome was analyzed without adjustment for the comorbidity, and this was another limitation of the present study. 


\section{Materials and Methods}

\subsection{Study Patients and Tissue Samples}

From January 2008 to April 2013, patients who underwent colonoscopy at National Taiwan University Hospital for screening, surveillance, or symptoms, and received endoscopic treatment or surgery, were included prospectively. The specimens in the discovery and validation set were collected consecutively by time sequence. Participants who had a history of inflammatory bowel disease or hereditary CRC, such as Lynch syndrome, familial adenomatous polyposis, or hyperplastic polyposis were excluded. Participants having active malignancy within five years before their diagnosis of CRC were also excluded. The specimens used for the discovery and validation of depressed neoplasm-specific biomarkers (D-marker panel) were FFPE tissues stored in the Department of Pathology, National Taiwan University Hospital. The specimens for testing survival outcome were fresh frozen CRC tissues, which were stored in liquid nitrogen. Before initiation, this study received approval (no. 201712033RINC) from the Institutional Review Board and Ethical Committee of the National Taiwan University Hospital.

\subsection{Classification of Colorectal Neoplasm Morphology}

According to the Paris classification, superficial colorectal neoplasms were morphologically categorized into depressed and polypoid [34]. The depressed neoplasm appeared slightly depressed on the surface, and the morphological classification was confirmed by experienced endoscopists using dye-based chromoendoscopy [35]. According to location, colorectal neoplasms were categorized as proximal (from the cecum to splenic flexure) and distal (from the descending colon to the rectum).

\subsection{Histological Diagnosis}

One experienced gastrointestinal pathologist (C.T. Shun) evaluated the histology of colorectal neoplasms according to the classification of the World Health Organization [36]. Advanced histology was defined by the presence of high-grade dysplasia, carcinoma in situ, or invasive cancer within the submucosa. Well/moderate differentiation and poor differentiation/undifferentiation were defined as low-grade and high-grade differentiation, respectively. CRCs invading the muscularis propria or beyond were not included for the development of the D-marker panel, as specifying their morphology was usually difficult. Moreover, serrated polyps were not included in the discovery and validation experiments, because these lesions progress to CRC through an alternative serrated pathway.

\subsection{OncoScan FFPE Array}

A total of $80 \mathrm{ng}$ genomic DNA was used for the OncoScan FFPE array (Affymetrix/Thermo Fisher Scientific, Waltham, MA, USA). The OncoScan FFPE array provides a molecular inversion probe (MIP) technology to identify CNAs, loss of heterozygosity, and somatic mutations. The OncoScan FFPE arrays were set up according to the OncoScan sample preparation instruction. Briefly, DNA samples were mixed with MIPs and incubated for 16-18 h for the annealing of genomic DNAs and probes. After incubation, exonuclease was added to remove free probes and genomic DNAs. MIPs were then linearized by using a restriction enzyme and amplified through polymerase chain reaction (PCR). The resulting products were hybridized to the OncoScan array for 16-18 h. Arrays were stained and washed in the GeneChip Fluidics Station 450 (Affymetrix/Thermo Fisher Scientific, Waltham, MA, USA) and loaded onto the GeneChip Scanner 3000 7G (Affymetrix/Thermo Fisher Scientific, Waltham, MA, USA). The intensity of fluorescence was scanned to generate array images. The raw results were further analyzed using Nexus Copy Number software (BioDiscovery, El Segundo, CA, USA), following the minimum information about a microarray experiment guidelines. 


\subsection{Droplet Digital PCR}

Genomic DNAs were first digested with EcoRI and HindIII for $1 \mathrm{~h}$. In total, $30 \mathrm{ng}$ digested genomic DNA was directly analyzed through droplet digital PCR (ddPCR). DdPCR was performed using the QX200 droplet digital PCR system (Bio-Rad, Hercules, CA, USA). Briefly, Taq polymerase PCR mixtures were assembled with a specific TaqMan probe, master mix, and digested genomic DNA samples. DG8 cartridges were loaded with $20 \mu \mathrm{L}$ PCR mixtures and $70 \mu \mathrm{L}$ droplet generation oil for each sample. The cartridges were placed into a droplet generator for emulsification, and the emulsified samples were then transferred onto a 96-well droplet PCR plate for 40 cycles of PCR. After PCR, the PCR plates were loaded into a droplet reader, which sequentially reads droplets from each well of the plate. Analysis of digital PCR data was performed using the RED mode of QX200 analysis software (version 1.2.10.0, Bio-Rad, Hercules, CA, USA).

\subsection{Molecular Subtype of CRC}

DNAs extracted from tissue specimens stored in liquid nitrogen were used for molecular marker testing. MSI was analyzed using the microsatellite instability analysis system (MSI Multiplex System Version 1.2, Promega, Madison, WI, USA), consisting of five quasi-monomorphic mononucleotide markers (BAT-25, BAT-26, NR-21, NR-24, and MONO-27). PCR products were separated by ABI 3730 Genetic Analyzer (Thermo Fisher Scientific, Waltham, MA, USA) and analyzed by GeneMapper 4.0 software (Thermo Fisher Scientific, Waltham, MA, USA). An internal lane size standard was added into the PCR samples to accurately size alleles and to adjust for run-to-run variations. The sample having two or more unstable markers were classified as MSI-high (MSI-H); otherwise, the patients were classified as microsatellite instable-low/microsatellite stable (MSI-L/MSS). The CIMP was determined by the panel (CACNA1G, IGF2, NEUROG1, RUNX3, and SOCS1). For methylation-specific PCR (MSP), 300 ng DNA were bisulfide-converted using EZ DNA Methylation-Gold Kits (ZYMO Research, Irvine, CA, USA) followed by 10 MSP reactions for each sample. All PCR reactions were performed with Human Methylated and Non-methylated DNA sets (ZYMO Research, Irvine, CA, USA) as positive and negative controls, respectively. Detection of MSP products were performed using the QIAxcel Advanced System (Qiagen, Hilden, Germany) through the run method, based on the instrument settings of AM $320 \mathrm{bp}$, AM $15 \mathrm{bp}-3 \mathrm{~kb}$, and SM 50-800 bp. If three or more methylated markers were identified, the sample was classified as CIMP-positive, and otherwise, the sample was classified as CIMP-negative. Molecular analysis for BRAF V600E/K/D and KRAS G12/13 mutations was performed with massARRAY (Agena, San Diego, CA, USA) with a multiplex test, as described previously [37,38].

\subsection{Statistics and Reproducibility}

Continuous and categorical variables were examined with Student's $t$ - and chi-square tests, respectively. The existence of gain/loss of chromosome regions was examined using Fisher's exact test. The survival outcome was tested through Kaplan-Meier analysis. The Cox regression was used for identifying the risk factor for cancer progression. Multivariable regression analysis was conducted to identify independent predictors of unfavorable survival. The risk of cancer progression contributed by a positive D-marker panel was adjusted by relevant risk factors without (model 1) and with cancer stage (model 2), respectively. In model 1, the positivity of the D-marker panel was adjusted according to age, gender, cancer differentiation, KRAS and BRAF mutations, CIMP-positivity, and MSI-H. In model 2 , the positivity of the D-marker panel was adjusted according to age, gender, cancer stage, cancer differentiation, KRAS and BRAF mutations, CIMP-positivity, and MSI-H. All tests were two-sided, and $p$ values less than 0.05 were considered statistically significant. Analyses were performed using $\mathrm{R}$ statistical software. 


\section{Conclusions}

Depressed neoplasms seem to arise via a distinct molecular pathway, in which alterations in MYC, $C C N A 1$, and BIRC7 play a significant role. These CNAs could not only stratify the risk of recurrence as biomarkers, but also predict the response to chemotherapy. This D-marker panel will improve our understanding of the pathogenesis of $\mathrm{CRC}$, and help to design tailored strategies for treatment and surveillance in order to optimize the overall effectiveness of CRC treatment, especially in proximal CRC. However, the current results remain preliminary, and further validation in prospective trials is warranted in the future.

Supplementary Materials: The following are available online at http:/www.mdpi.com/2072-6694/12/6/1527/s1, Figure S1: Depressed neoplasm has invasive cancer at small-size stage, Figure S2: Copy number alterations in depressed and polypoid neoplasms, Figure S3: Arm-level changes in MYC, CCNA1, and BIRC7 in each case in the discovery set, Table S1: Matched protein coding genes with significant arm-level changes in chr8, chr13, and chr20, Table S2: Diagnostic performance of the D-mark panel for detecting depressed neoplasms.

Author Contributions: The study was conceived by L.-C.C. and H.-M.C., with input from S.-L.Y. and M.-S.W. M.-S.W., S.-L.Y., and J.-T.L. are the guarantors of the article. L.-C.C. designed the study and wrote the protocol. H.-M.C., C.T.S., and J.-T.L. recruited patients to the study. J.-T.L., H.-M.C., C.-T.S., B.-C.H., M.-H.C., Y.-C.H., W.-T.C., and K.-Y.S. generated, collected, analyzed, and interpreted the data. L.-C.C., H.-M.C., S.-L.Y., and M.-S.W. drafted and revised the manuscript. All authors approved the final version of the manuscript. All authors have read and agreed to the published version of the manuscript.

Funding: The study was funded by the National Taiwan University Hospital (grant numbers: NTUH 107-M3990, NTUH 108-M4209); the Ministry of Science and Technology, Taiwan (grant numbers: MOST-105-2314-B-002-047, MOST106-2319-B-002-002, and MOST107-2319-B-002-002); and the "Center of Precision Medicine" from The Featured Areas Research Center Program, within the framework of the Higher Education Sprout Project by the Ministry of Education (MOE) in Taiwan.

Acknowledgments: We thank the Pharmacogenomics Lab of the National Core Facility for biotechnology and technical support.

Conflicts of Interest: The authors declare no conflicts of interest.

\section{Abbreviations}

$\begin{array}{ll}\text { CRC } & \text { colorectal cancer } \\ \text { CAN } & \text { copy number alteration } \\ \text { FFPE } & \text { formalin-fixed paraffin-embedded } \\ \text { MIP } & \text { molecular inversion probe } \\ \text { ddPCR } & \text { droplet digital polymerase chain reaction } \\ \text { MSI } & \text { microsatellite instability } \\ \text { CIMP } & \text { CpG island methylation phenotype } \\ \text { MSP } & \text { methylation-specific PCR } \\ \text { PFS } & \text { progression-free survival } \\ \text { OS } & \text { overall survival } \\ \text { HR } & \text { hazard ratio } \\ \text { LNM } & \text { lymph node metastasis }\end{array}$

\section{References}

1. Muto, T.; Kamiya, J.; Sawada, T.; Konoshi, F.; Sugihara, K.; Kubota, Y.; Adachi, M.; Agawa, S.; Saito, Y.; Morioka, Y.; et al. Small "flat adenoma" of the large bowel with special reference to its clinicopathologic features. Dis. Colon Rectum 1985, 28, 847-851. [CrossRef] [PubMed]

2. Rembacken, B.; Fujii, T.; Cairns, A.; Dixon, M.F.; Yoshida, S.; Chalmers, D.M.; Axon, A.T. Flat and depressed colonic neoplasms: A prospective study of 1000 colonoscopies in the UK. Lancet 2000, 355, 1211-1214. [CrossRef]

3. Chiu, H.M.; Lin, J.T.; Chen, C.C.; Lee, Y.C.; Liao, W.C.; Liang, J.T.; Shun, C.T.; Wang, H.P.; Wu, M.S. Prevalence and characteristics of nonpolypoid colorectal neoplasm in an asymptomatic and average-risk Chinese population. Clin. Gastroenterol. Hepatol. 2009, 7, 463-470. [CrossRef] [PubMed] 
4. Tsuda, S.; Veress, B.; Toth, E.; Fork, F.T. Flat and depressed colorectal tumours in a southern Swedish population: A prospective chromoendoscopic and histopathological study. Gut 2002, 51, 550-555. [CrossRef] [PubMed]

5. Saitoh, Y.; Waxman, I.; West, A.B.; Popnikolov, N.K.; Gatalica, Z.; Watari, J.; Obara, T.; Kohgo, Y.; Pasricha, P.J. Prevalence and distinctive biologic features of flat colorectal adenomas in a North American population. Gastroenterology 2001, 120, 1657-1665. [CrossRef] [PubMed]

6. Parra-Blanco, A.; Gimeno-Garcia, A.Z.; Nicolas-Perez, D.; Garcia, C.; Medina, C.; Diaz-Flores, L.; Grosso, B.; Jimenez, A.; Quintero, E. Risk for high-grade dysplasia or invasive carcinoma in colorectal flat adenomas in a Spanish population. Gastroenterol. Hepatol. 2006, 29, 602-609. [CrossRef]

7. Kudo, S. Endoscopic mucosal resection of flat and depressed types of early colorectal cancer. Endoscopy 1993, 25, 455-461. [CrossRef] [PubMed]

8. Matsuda, T.; Saito, Y.; Hotta, K.; Sano, Y.; Fujii, T. Prevalence and clinicopathological features of nonpolypoid colorectal neoplasms: Should we pay more attention to identifying flat and depressed lesions? Dig. Endosc. 2010, 22 (Suppl. 1), S57-S62. [CrossRef] [PubMed]

9. Chang, L.C.; Chiu, H.M.; Shun, C.T.; Liang, J.T.; Lin, J.T.; Chen, C.C.; Lee, Y.C.; Wu, M.S. Mutational profiles of different macroscopic subtypes of colorectal adenoma reveal distinct pathogenetic roles for KRAS, BRAF and PIK3CA. BMC Gastroenterol. 2014, 14, 221. [CrossRef] [PubMed]

10. Voorham, Q.J.M.; Rondagh, E.J.A.; Knol, D.L.; van Engeland, M.; Carvalho, B.; Meijer, G.A.; Sanduleanu, S. Tracking the molecular features of nonpolypoid colorectal neoplasms: A systematic review and meta-analysis. Am. J. Gastroenterol. 2013, 108, 1042-1056. [CrossRef] [PubMed]

11. Benedix, F.; Kube, R.; Meyer, F.; Schmidt, U.; Gastinger, I.; Lippert, H. Comparison of 17,641 patients with right- and left-sided colon cancer: Differences in epidemiology, perioperative course, histology, and survival. Dis. Colon Rectum 2010, 53, 57-64. [CrossRef] [PubMed]

12. Meguid, R.A.; Slidell, M.B.; Wolfgang, C.L.; Chang, D.C.; Ahuja, N. Is there a difference in survival between right- versus left-sided colon cancers? Ann. Surg. Oncol. 2008, 15, 2388-2394. [CrossRef] [PubMed]

13. Weiss, J.M.; Pfau, P.R.; O'Connor, E.S.; King, J.; LoConte, N.; Kennedy, G.; Smith, M.A. Mortality by stage for right- versus left-sided colon cancer: Analysis of surveillance, epidemiology, and end results-Medicare data. J. Clin. Oncol. 2011, 29, 4401-4409. [CrossRef] [PubMed]

14. Sinicrope, F.; Mahoney, M.R.; Smyrk, T.C.; Thibodeau, S.N.; Warren, R.S.; Bertagnolli, M.M.; Nelson, G.D.; Goldberg, R.M.; Sargent, D.J.; Alberts, S.R. Prognostic impact of deficient DNA mismatch repair in patients with stage III colon cancer from a randomized trial of FOLFOX-based adjuvant chemotherapy. J. Clin. Oncol. 2013, 31, 3664-3672. [CrossRef] [PubMed]

15. Brulé, S.Y.; Jonker, D.J.; Karapetis, C.S.; O'Callaghan, C.J.; Moore, M.J.; Wong, R.; Tebbutt, N.C.; Underhill, C.; Yip, D.; Zalcberg, J.R.; et al. Location of colon cancer (right-sided versus left-sided) as a prognostic factor and a predictor of benefit from cetuximab in NCIC CO.17. Eur. J. Cancer 2015, 51, 1405-1414. [CrossRef] [PubMed]

16. Han, X.; Tan, Q.; Yang, S.; Li, J.; Xu, J.; Hao, X.; Hu, X.; Xing, P.; Liu, Y.; Lin, L.; et al. Comprehensive Profiling of Gene Copy Number Alterations Predicts Patient Prognosis in Resected Stages I-III Lung Adenocarcinoma. Front. Oncol. 2019, 9, 556. [CrossRef] [PubMed]

17. Rennstam, K.; Ahlstedt-Soini, M.; Baldetorp, B.; Bendahl, P.O.; Borg, A.; Karhu, R.; Tanner, M.; Tirkkonen, M.; Isola, J. Patterns of chromosomal imbalances defines subgroups of breast cancer with distinct clinical features and prognosis. A study of 305 tumors by comparative genomic hybridization. Cancer Res. 2003, 63, 8861-8868. [PubMed]

18. Yu, Y.; Cao, J.; Wu, W.; Zhu, Q.; Tang, Y.; Zhu, C.; Dai, J.; Li, Z.; Wang, J.; Xue, L.; et al. Genome-wide copy number variation analysis identified ANO1 as a novel oncogene and prognostic biomarker in esophageal squamous cell cancer. Carcinogenesis 2019, 40, 1198-1208. [CrossRef] [PubMed]

19. Vasaikar, S.; Huang, C.; Wang, X.; Petyuk, V.A.; Savage, S.R.; Wen, B.; Dou, Y.; Zhang, Y.; Shi, Z.; Arshad, O.A.; et al. Proteogenomic Analysis of Human Colon Cancer Reveals New Therapeutic Opportunities. Cell 2019, 177, 1035-1049.e19. [CrossRef] [PubMed]

20. Zhang, B.; Wang, J.; Wang, X.; Zhu, J.; Liu, Q.; Shi, Z.; Chambers, M.C.; Zimmerman, L.J.; Shaddox, K.F.; Kim, S.; et al. Proteogenomic characterization of human colon and rectal cancer. Nature 2014, 513, 382-387. [CrossRef] [PubMed] 
21. He, T.C.; Sparks, A.B.; Rago, C.; Hermeking, H.; Zawel, L.; da Costa, L.T.; Morin, P.J.; Vogelstein, B.; Kinzler, K.W. Identification of c-MYC as a target of the APC pathway. Science 1998, 281, 1509-1512. [CrossRef] [PubMed]

22. Sansom, O.J.; Meniel, V.S.; Muncan, V.; Phesse, T.J.; Wilkins, J.A.; Reed, K.R.; Vass, J.K.; Athineos, D.; Clevers, H.; Clarke, A.R. Myc deletion rescues Apc deficiency in the small intestine. Nature 2007, 446, 676-679. [CrossRef] [PubMed]

23. Wegiel, B.; Bjartell, A.; Tuomela, J.; Dizeyi, N.; Tinzl, M.; Helczynski, L.; Nilsson, E.; Otterbein, L.E.; Harkonen, P.; Persson, J.L. Multiple cellular mechanisms related to cyclin A1 in prostate cancer invasion and metastasis. J. Natl. Cancer Inst. 2008, 100, 1022-1036. [CrossRef] [PubMed]

24. Ge, Y.; Cao, X.; Wang, D.; Sun, W.; Sun, H.; Han, B.; Cui, J.; Liu, B. Overexpression of Livin promotes migration and invasion of colorectal cancer cells by induction of epithelial-mesenchymal transition via NF-kappaB activation. Onco Targets Ther. 2016, 9, 1011-1021. [PubMed]

25. Goto, H.; Oda, Y.; Murakami, Y.; Tanaka, T.; Hasuda, K.; Goto, S.; Sasaki, Y.; Sakisaka, S.; Hattori, M. Proportion of de novo cancers among colorectal cancers in Japan. Gastroenterology 2006, 131, 40-46. [CrossRef] [PubMed]

26. Chen, C.D.; Yen, M.F.; Wang, W.M.; Wong, J.M.; Chen, T.H. A case-cohort study for the disease natural history of adenoma-carcinoma and de novo carcinoma and surveillance of colon and rectum after polypectomy: Implication for efficacy of colonoscopy. Br. J. Cancer 2003, 88, 1866-1873. [CrossRef] [PubMed]

27. Bosch, S.L.; Teerenstra, S.; De Wilt, J.H.; Cunningham, C.; Nagtegaal, I.D. Predicting lymph node metastasis in pT1 colorectal cancer: A systematic review of risk factors providing rationale for therapy decisions. Endoscopy 2013, 45, 827-834. [CrossRef] [PubMed]

28. Ikematsu, H.; Yoda, Y.; Matsuda, T.; Yamaguchi, Y.; Hotta, K.; Kobayashi, N.; Fujii, T.; Oono, Y.; Sakamoto, T.; Nakajima, T.; et al. Long-term outcomes after resection for submucosal invasive colorectal cancers. Gastroenterology 2013, 144, 551-559.e14. [CrossRef] [PubMed]

29. Ozawa, T.; Kandimalla, R.; Gao, F.; Nozawa, H.; Hata, K.; Nagata, H.; Okada, S.; Izumi, D.; Baba, H.; Fleshman, J.; et al. A MicroRNA Signature Associated With Metastasis of T1 Colorectal Cancers to Lymph Nodes. Gastroenterology 2018, 154, 844-848.e7. [CrossRef] [PubMed]

30. Dang, C.V. MYC on the path to cancer. Cell 2012, 149, 22-35. [CrossRef]

31. Liu, H.; Wang, S.; Sun, H.; Pan, Z.; Zhou, W.; Wu, M. Inhibition of tumorigenesis and invasion of hepatocellular carcinoma by siRNA-mediated silencing of the livin gene. Mol. Med. Rep. 2010, 3, 903-907. [PubMed]

32. De La Chapelle, A.; Hampel, H. Clinical relevance of microsatellite instability in colorectal cancer. J. Clin. Oncol. 2010, 28, 3380-3387. [CrossRef] [PubMed]

33. Jaramillo, E.; Watanabe, M.; Slezak, P.; Rubio, C. Flat neoplastic lesions of the colon and rectum detected by high-resolution video endoscopy and chromoscopy. Gastrointest. Endosc. 1995, 42, 114-122. [CrossRef]

34. The Paris endoscopic classification of superficial neoplastic lesions: Esophagus, stomach, and colon: November 30 to December 1, 2002. Gastrointest. Endosc. 2003, 58, S3-S43. [CrossRef]

35. Kudo, S.E.; Lambert, R.; Allen, J.I.; Fujii, H.; Fujii, T.; Kashida, H.; Matsuda, T.; Mori, M.; Saito, H.; Shimoda, T.; et al. Nonpolypoid neoplastic lesions of the colorectal mucosa. Gastrointest. Endosc. 2008, 68, S3-S47. [CrossRef] [PubMed]

36. Bosman, F.T.; Carneiro, F.; Hruban, R.H.; Neil, D. Pathology and Genetics of Tumors of the Digestive System; International Agency for Research on Cancer Press: Lyon, France, 2000.

37. Hsu, K.H.; Ho, C.C.; Hsia, T.C.; Tseng, J.S.; Su, K.Y.; Wu, M.F.; Chiu, K.L.; Yang, T.Y.; Chen, K.C.; Ooi, H.; et al. Identification of five driver gene mutations in patients with treatment-naive lung adenocarcinoma in Taiwan. PLoS ONE 2015, 10, e0120852. [CrossRef] [PubMed]

38. Lin, C.C.; Shih, J.Y.; Yu, C.J.; Ho, C.C.; Liao, W.Y.; Lee, J.H.; Tsai, T.H.; Su, K.Y.; Hsieh, M.S.; Chang, Y.L.; et al. Outcomes in patients with non-small-cell lung cancer and acquired Thr790Met mutation treated with osimertinib: A genomic study. Lancet Respir. Med. 2018, 6, 107-116. [CrossRef]

(C) 2020 by the authors. Licensee MDPI, Basel, Switzerland. This article is an open access article distributed under the terms and conditions of the Creative Commons Attribution (CC BY) license (http://creativecommons.org/licenses/by/4.0/). 